\title{
IMPACT OF SELECTED DETERMINANTS OF CORPORATE GOVERNANCE ON FINANCIAL PERFORMANCE OF COMPANIES
}

\author{
Janka Grofcikova ${ }^{1, a, *}$ \\ ${ }^{1}$ Faculty of Ecomonics, Matej Bel University in Banska Bystrica, Department of Finance and \\ Accounting, Tajovského 10, Banská Bystrica, Slovakia \\ ajanka.grofcikova@umb.sk \\ *Corresponding author
}

Cite as: Grofcikova, J. (2020). Impact of selected determinants of corporate governance on financial performance of companies, Ekonomicko-manazerske spektrum, 14(2), 12-23.

Available at: dx.doi.org/10.26552/ems.2020.2.12-23.

Received: 24 March 2020; Received in revised form: 12 June 2020; Accepted: 20 July 2020; Available online: 24 August 2020

\begin{abstract}
The role of corporate governance (CG) is to ensure functioning of companies in accordance with their formulated objectives to ensure growth of corporate assets and satisfaction of the owners. In addition to management of the company, there are other stakeholders whose interests need to be considered in meeting the owners' objectives. These include creditors, employees, clients, and the wider context of the business. The aim of this paper is to explore and compare the impact of selected financial and non-financial determinants representing the interests of these groups on corporate financial performance. The influence of determinants of CG on financial performance, measured by return on assets (ROA), return on equity (ROE) and return on sales (ROS) indicators, is investigated by means of correlation analysis. The sample of enterprises used consists of non-financial joint-stock companies listed on the Bratislava Stock Exchange, insurance companies, and banks based in Slovakia. The findings show that each of the investigated determinants of CG affects financial performance of companies. ROA, ROE and ROS of share issuers are significantly influenced by the total equity (EQ), average remuneration (AR) and number of the Board of Supervisor members (BSM). With banks, performance indicators are only influenced by total personal costs (PC). ROA, ROE and ROS of all companies are influenced by the dividend ratio (DR), EQ, AR and BSM.
\end{abstract}

Keywords: corporate governance, globalization, financial performance, stakeholders' interests JEL Classification: F65, G32, G34

\section{Introduction}

Corporate governance $(\mathrm{CG})$ is currently the focus of several researches. The role of $\mathrm{CG}$ is to ensure functioning of companies in accordance with their formulated strategic and operational objectives, which are to ensure the growth of value of the company's assets and satisfaction of its owners. The principles recommended in CG are the result of globalization trends (Schymik, 2018) and have a transnational dimension.

Several important organizations have been dealing with CG issues. Among them are the Organisation for Economic Co-operation and Development (OECD). According to OECD, CG 
helps to build the climate of confidence, transparency, and accountability needed to support longterm investments, financial stability, and corporate integrity, thereby fostering stronger growth and a more inclusive society (www.oecd.org). In order to fulfil its mission, the OECD drafted CG principles in 1999 and reviewed and supplemented them in 2004 and 2015. The current version was developed in cooperation of the OECD Corporate Governance Committee and the G20. The document consists of 6 separate chapters: (I) Ensuring the basis for an effective CG framework, (II) The rights and equitable treatment of shareholders and key ownership functions, (III) Institutional investors, stock markets, and other intermediaries, (IV) The role of stakeholders in CG, (V) Disclosure and transparency, (VI) The responsibilities of the board. Each of the chapters contains a list of supporting sub-principles, supplemented by explanatory notes.

Relationships between corporate performance and CG are discussed in detail by the experts in the field and many contributions have been published on the topic (e.g., Bhagat and Bolton, 2008; Bhagat and Bolton, 2019; Paniagua et al., 2018; Di Berardino, 2015; Naciti, 2019; Hearn, 2011).

Khanchel (2007) examines CG issues from the perspective of its measurement and proposes the following determinants of strong CG: independent directors, independence of committees, board size, split chairman/CEO roles, board meetings, competence of audit committee members, reputation of auditors, audit committee meetings.

A well-functioning business management system helps the company attract investment, raise funds (Musa et al., 2014b) and strengthen key business performance factors (Grofcikova, 2016a; Grofcikova, 2016b; Valaskova et al., 2018; Rodriguez-Fernandez, 2016). In addition, good CG strengthens financial stability of the company and its resilience to future financial problems (Musa et al., 2014a), improves the decision-making processes and reduces conflicts of interest between stakeholders, and minimizes shareholder control over company management. This, according to Shleifer and Vishny (1997), increases the likelihood that managers will invest in projects with a positive net present value.

Zahroh and Hamidah (2016) examine the relationship between corporate financial performance and CG in terms of board size and independence, audit committee independence, audit quality, and the degree of implementation of CG principles. Other contributions examine the impact of selected determinants of CG on corporate performance, measured, most commonly by ROA and ROE, on samples of enterprises from Vietnam (Vo and Phan, 2013), Malaysia (Fooladi et al., 2014), India (Waleed et al., 2019), Singapore (Vu and Nguyen, 2017), Japan (Mizuno, 2010), Turkey (Ararat et al., 2017), Thailand (Detthamrong et al., 2017), or the United Kingdom (Akbar et al., 2016).

CG models are based on basic management theories that Afza and Nazir (2014) categorize as agency theory, stewardship theory, stakeholder theory, resource dependence theory, transaction cost theory, and political theory.

The contribution is based on the assumptions of the stakeholder theory, because in addition to the management of the business, other groups of people whose interests need to be considered are involved in meeting the objectives of the owners. These include creditors who provide repayable funding to the enterprise, employees, clients, but also the wider environment of the enterprise represented by the state and the population.

\section{Methodology}

The aim of the paper is to present the results of the research intended to examine and compare the impact of selected financial and non-financial determinants representing the interests of four basic interest groups in accordance with the stakeholder theory on companies' financial performance. The researcher measured the companies' performance based on the indicators of 
return on assets, return on equity and return on sales, calculated from the net profit of each given company. The research was conducted on a basic set of entities, divided into three groups: (1) non-financial joint-stock companies listed on the Bratislava Stock Exchange, (2) insurance companies based in Slovakia and (3) banks and home savings banks based in Slovakia. These are all joint-stock companies, and as such must under the valid legislation of the Slovak Republic set up their corporate governing bodies, e.g., the board of directors and the board of supervisors, which as determinants of CG are part of focus of the present research. For the same reason, branches of foreign banks and insurance companies were not considered. The objective of the research was to compare the findings between the defined groups of these subjects.

The descriptions and definitions of selected financial and non-financial determinants of CG, including identification of the interest group that the determinant represents, are included in Table 1.

Table 1: Definition of variables examined

\begin{tabular}{lll}
\hline & Symbol & Description and measurement (stakeholders identifier) \\
\hline & Return on Assets in \% (net profit / total assets) \\
Return on Equity in \% (net profit / equity) \\
Return on Settlement in \% (net profit / revenues) \\
Total equity (in EUR) (1) \\
Dividend ratio in \% (dividend paid in 2017 / net profit in 2016) (1) \\
Average remuneration per member of the Company's governing bodies (in EUR) (total \\
DR
\end{tabular}

Stakeholder identifier: (1) shareholders, (2) management, (3) employees, (4) creditors.

Source: own processing

The aim was to examine the interests of business owners using the size of the company's equity, dividend per share, percentage of the registered capital held by the largest shareholder, and the number of shareholders with qualifying holdings in the registered capital. The company's top management is represented by the indicator of average annual remuneration per member of the board and number of members of the board of directors and the board of supervisors. Employees' interests are considered by including their total number, middle management share, and annual personnel costs in the variable group surveyed. Creditors' interests are represented by the indicator of total indebtedness.

The data were drawn from the annual reports of individual companies for 2017 , as available on the companies' websites.

The influence of selected determinants of CG on the financial performance of companies is investigated by means of a correlation analyses, namely Pearson's R, Spearman correlation, and Somers' D, which measure unidirectional dependence of variables. Dependent variables are indicators of financial performance; independent variables are individual determinants of CG. Hypotheses about the existence of dependence between the dependent and independent variable $\left(\mathrm{H}_{0}: \rho=0 ; \mathrm{H}_{1}: \rho \neq 0\right)$ are verified at the significance level $\alpha=0.1$. 
Table 2: Correlation analysis

\begin{tabular}{|c|c|c|c|c|c|c|c|c|c|c|c|c|c|}
\hline \multicolumn{2}{|c|}{ Dependent variable } & \multicolumn{2}{|l|}{ ROA } & \multicolumn{2}{|l|}{ ROE } & \multicolumn{2}{|l|}{ ROS } & \multicolumn{2}{|l|}{ ROA } & \multicolumn{2}{|l|}{ ROE } & \multicolumn{2}{|l|}{ ROS } \\
\hline \multicolumn{14}{|c|}{ Non-financial determinants of corporate governance } \\
\hline \multicolumn{2}{|c|}{ Independent variable } & \multicolumn{6}{|l|}{ LHS } & \multicolumn{6}{|l|}{ BSM } \\
\hline \multirow[t]{2}{*}{ Entities } & $\begin{array}{l}\text { Stat. } \\
\text { indicator }\end{array}$ & Value & Sig. & Value & Sig. & Value & Sig. & Value & Sig. & Value & Sig. & Value & Sig. \\
\hline & $\begin{array}{l}\text { Pearson's } \\
\text { R }\end{array}$ & -.088 & .786 & .100 & .757 & -.120 & .710 & -.013 & .969 & -.232 & .467 & -.004 & .991 \\
\hline \multirow[t]{3}{*}{ Banks } & $\begin{array}{l}\text { Spearman } \\
\text { Corr. }\end{array}$ & -.112 & .729 & -.034 & .917 & -.269 & .398 & .152 & .637 & -.081 & .802 & .230 & .473 \\
\hline & Somers' d & -.098 & .679 & -.020 & .940 & -.216 & .375 & .016 & .936 & -.082 & .672 & .148 & .428 \\
\hline & $\begin{array}{l}\text { Pearson's } \\
\text { R }\end{array}$ & .125 & .510 & -.223 & .236 & -.086 & .650 & .276 & .114 & .174 & .325 & .162 & .361 \\
\hline \multirow[t]{3}{*}{$\begin{array}{l}\text { Issuers of } \\
\text { shares }\end{array}$} & $\begin{array}{l}\text { Spearman } \\
\text { Corr. }\end{array}$ & -.034 & .856 & -.158 & .404 & -.218 & .247 & $.381^{* *}$ & .026 & $.423^{* *}$ & .013 & .231 & .188 \\
\hline & Somers' d & -.039 & .782 & -.103 & .486 & -.168 & .202 & $.451^{* *}$ & .016 & $.487^{* * * *}$ & .007 & $.278^{*}$ & .096 \\
\hline & $\begin{array}{l}\text { Pearson's } \\
\text { R }\end{array}$ & .093 & .752 & .217 & .455 & .293 & .310 & -.127 & .651 & .071 & .801 & -.089 & .752 \\
\hline \multirow[t]{3}{*}{$\begin{array}{l}\text { Insurance } \\
\text { companies }\end{array}$} & $\begin{array}{l}\text { Spearman } \\
\text { Corr. }\end{array}$ & .169 & .565 & .155 & .597 & .308 & .284 & .069 & .808 & .039 & .890 & -.183 & .513 \\
\hline & Somers' d & .160 & .471 & .136 & .466 & .235 & .227 & .070 & .747 & .000 & 1.000 & -.116 & .407 \\
\hline & $\begin{array}{l}\text { Pearson's } \\
\text { R }\end{array}$ & $.209^{* *}$ & .012 & .047 & .730 & .163 & .230 & .172 & .186 & .162 & .212 & .180 & .164 \\
\hline \multirow[t]{2}{*}{$\begin{array}{l}\text { All } \\
\text { entities }\end{array}$} & $\begin{array}{l}\text { Spearman } \\
\text { Corr. }\end{array}$ & .061 & .655 & $.298^{* *}$ & .026 & .199 & .141 & $.215^{*}$ & .096 & $.343^{* * * *}$ & .007 & $.266^{* *}$ & .038 \\
\hline & Somers' d & .038 & .666 & $.208^{* *}$ & .025 & .141 & .132 & $.195^{*}$ & .054 & $.301^{* * *}$ & .003 & $.229^{* *}$ & .014 \\
\hline \multicolumn{2}{|c|}{ Independent variable } & QSH & & & & & & EMP & & & & & \\
\hline \multirow{3}{*}{ Banks } & $\begin{array}{l}\text { Pearson's } \\
\text { R }\end{array}$ & -.138 & .669 & -.152 & .638 & .064 & .844 & .393 & .207 & .301 & .342 & .346 & .270 \\
\hline & $\begin{array}{l}\text { Spearman } \\
\text { Corr. }\end{array}$ & -.101 & .755 & -.101 & .755 & .073 & .821 & .364 & .245 & $.552^{*}$ & .063 & .462 & .131 \\
\hline & Somers' d & -.133 & .722 & -.200 & .540 & .067 & .826 & .242 & .168 & $.424^{* *}$ & .019 & $.333^{*}$ & .100 \\
\hline \multirow{3}{*}{$\begin{array}{l}\text { Issuers of } \\
\text { shares }\end{array}$} & $\begin{array}{l}\text { Pearson's } \\
\text { R }\end{array}$ & .220 & .219 & .276 & .119 & -.016 & .931 & .176 & .318 & .074 & .679 & .095 & .594 \\
\hline & $\begin{array}{l}\text { Spearman } \\
\text { Corr. }\end{array}$ & .181 & .314 & .176 & .328 & .256 & .150 & .165 & .351 & .219 & .214 & .029 & .872 \\
\hline & Somers' d & .158 & .300 & .144 & .348 & .219 & .143 & .103 & .401 & .139 & .265 & .038 & .800 \\
\hline \multirow{3}{*}{$\begin{array}{l}\text { Insurance } \\
\text { companies }\end{array}$} & $\begin{array}{l}\text { Pearson's } \\
\text { R }\end{array}$ & -.119 & .686 & -.045 & .880 & .081 & .782 & -.131 & .641 & .174 & .535 & .013 & .964 \\
\hline & $\begin{array}{l}\text { Spearman } \\
\text { Corr. }\end{array}$ & -.135 & .645 & -.066 & .822 & -.103 & .726 & -.232 & .405 & -.189 & .499 & -.143 & .612 \\
\hline & Somers' d & -.127 & .644 & -.055 & .865 & -.091 & .724 & -.143 & .574 & -.143 & .565 & -.048 & .814 \\
\hline \multirow{3}{*}{$\begin{array}{l}\text { All } \\
\text { entities }\end{array}$} & $\begin{array}{l}\text { Pearson's } \\
\text { R }\end{array}$ & .074 & .576 & .082 & .538 & -.139 & .294 & .100 & .442 & .155 & .233 & $.272^{* *}$ & .034 \\
\hline & $\begin{array}{l}\text { Spearman } \\
\text { Corr. }\end{array}$ & .056 & .676 & -.159 & .229 & -.042 & .752 & .153 & .239 & $.386^{* * * *}$ & .002 & $.298^{* *}$ & .020 \\
\hline & Somers' d & .047 & .671 & -.135 & .214 & -.036 & .752 & .100 & .238 & $.261^{* * * *}$ & .002 & $.218^{* *}$ & .024 \\
\hline
\end{tabular}




\begin{tabular}{|c|c|c|c|c|c|c|c|c|c|c|c|c|c|}
\hline \multicolumn{2}{|c|}{ Independent variable } & \multicolumn{6}{|l|}{ BDM } & \multicolumn{6}{|l|}{ MAN } \\
\hline & $\begin{array}{l}\text { Pearson's } \\
\mathrm{R}\end{array}$ & .311 & .326 & .314 & .320 & .278 & .381 & .285 & .458 & $.597^{*}$ & .089 & .270 & .482 \\
\hline \multirow[t]{3}{*}{ Banks } & $\begin{array}{l}\text { Spearman } \\
\text { Corr. }\end{array}$ & .397 & .202 & .375 & .230 & .277 & .384 & .350 & .356 & .283 & .460 & .000 & 1.000 \\
\hline & Somers' d & .333 & .185 & .296 & .168 & .259 & .311 & .278 & .130 & .222 & .300 & .056 & .769 \\
\hline & $\begin{array}{l}\text { Pearson's } \\
\text { R }\end{array}$ & -.124 & .484 & .019 & .920 & .140 & .431 & -.077 & .669 & -.042 & .817 & -.096 & .595 \\
\hline \multirow[t]{3}{*}{$\begin{array}{l}\text { Issuers of } \\
\text { shares }\end{array}$} & $\begin{array}{l}\text { Spearman } \\
\text { Corr. }\end{array}$ & .086 & .629 & .109 & .540 & .107 & .549 & .051 & .779 & .079 & .661 & .002 & .993 \\
\hline & Somers' d & .088 & .610 & .111 & .529 & .094 & .533 & .050 & .710 & .069 & .579 & .022 & .891 \\
\hline & $\begin{array}{l}\text { Pearson's } \\
\mathrm{R}\end{array}$ & -.099 & .726 & -.092 & .745 & -.204 & .466 & .096 & .732 & -.306 & .268 & -.349 & .203 \\
\hline \multirow[t]{3}{*}{$\begin{array}{l}\text { Insurance } \\
\text { companies }\end{array}$} & $\begin{array}{l}\text { Spearman } \\
\text { Corr. }\end{array}$ & -.054 & .849 & -.295 & .286 & -.322 & .241 & -.121 & .666 & -.032 & .909 & -.132 & .639 \\
\hline & Somers' d & -.060 & .752 & -.277 & .217 & -.277 & .180 & -.086 & .720 & -.010 & .966 & -.067 & .769 \\
\hline & $\begin{array}{l}\text { Pearson's } \\
\text { R }\end{array}$ & -.003 & .979 & .132 & .311 & $.218^{*}$ & .092 & -.010 & .940 & -.006 & .965 & -.110 & .417 \\
\hline \multirow[t]{2}{*}{$\begin{array}{l}\text { All } \\
\text { entities }\end{array}$} & $\begin{array}{l}\text { Spearman } \\
\text { Corr. }\end{array}$ & .158 & .224 & $.276^{* *}$ & .031 & .175 & .178 & .172 & .200 & .167 & .215 & -.001 & .996 \\
\hline & Somers' d & .134 & .209 & $.250^{* * *}$ & .202 & .159 & .159 & .119 & .230 & .133 & .161 & .026 & .809 \\
\hline
\end{tabular}

Financial determinants of corporate governance

\begin{tabular}{|c|c|c|c|c|c|c|c|c|c|c|c|c|c|}
\hline \multirow{2}{*}{\multicolumn{2}{|c|}{$\begin{array}{l}\text { Dependent variable } \\
\text { Independent variable }\end{array}$}} & \multicolumn{2}{|l|}{ ROA } & \multicolumn{2}{|l|}{ ROE } & \multicolumn{2}{|l|}{ ROS } & \multicolumn{2}{|l|}{ ROA } & \multicolumn{2}{|l|}{ ROE } & \multicolumn{2}{|l|}{ ROS } \\
\hline & & EQ & & & & & & $\mathrm{AR}$ & & & & & \\
\hline Entities & $\begin{array}{l}\text { Stat. } \\
\text { indicator }\end{array}$ & Value & Sig. & Value & Sig. & Value & Sig. & Value & Sig. & Value & Sig. & Value & Sig. \\
\hline \multirow{3}{*}{ Banks } & $\begin{array}{l}\text { Pearson's } \\
\text { R }\end{array}$ & $.499^{*}$ & .098 & -.080 & .806 & .417 & .177 & .427 & .166 & .365 & .243 & .403 & .194 \\
\hline & $\begin{array}{l}\text { Spearman } \\
\text { Corr. }\end{array}$ & .483 & .112 & .252 & .430 & $.608^{* * *}$ & .036 & .378 & .226 & $.573^{*}$ & .051 & .399 & .199 \\
\hline & Somers' d & $.364^{* *}$ & .037 & .182 & .418 & $.515^{* * * *}$ & .009 & .242 & .256 & $.424^{* *}$ & .023 & .212 & .284 \\
\hline \multirow{3}{*}{$\begin{array}{l}\text { Issuers of } \\
\text { shares }\end{array}$} & $\begin{array}{l}\text { Pearson's } \\
\text { R }\end{array}$ & .149 & .400 & .075 & .672 & .053 & .766 & .173 & .335 & .084 & .644 & .116 & .521 \\
\hline & $\begin{array}{l}\text { Spearman } \\
\text { Corr. }\end{array}$ & $.422^{* *}$ & .013 & $.468^{* * *}$ & .005 & $.293^{*}$ & .093 & $.387^{* *}$ & .026 & $.409^{* * *}$ & .018 & $.297^{*}$ & .093 \\
\hline & Somers' d & $.294^{* *}$ & .013 & $.333^{* * *}$ & .005 & $.219^{*}$ & .070 & $.299^{* *}$ & .016 & $.320^{* *}$ & .016 & $.238^{*}$ & .087 \\
\hline \multirow{3}{*}{$\begin{array}{l}\text { Insurance } \\
\text { companies }\end{array}$} & $\begin{array}{l}\text { Pearson's } \\
\mathrm{R}\end{array}$ & -.057 & .839 & .220 & .430 & -.027 & .925 & -.107 & .703 & .270 & .331 & .110 & .697 \\
\hline & $\begin{array}{l}\text { Spearman } \\
\text { Corr. }\end{array}$ & -.096 & .732 & .021 & .940 & -.054 & .850 & -.107 & .704 & .018 & .950 & .007 & .980 \\
\hline & Somers' d & -.086 & .748 & -.010 & .969 & -.029 & .896 & .010 & .970 & .010 & .966 & .029 & .903 \\
\hline \multirow{3}{*}{$\begin{array}{l}\text { All } \\
\text { entities }\end{array}$} & $\begin{array}{l}\text { Pearson's } \\
\text { R }\end{array}$ & .108 & .409 & .106 & .416 & $.244^{*}$ & .058 & .152 & .247 & $.220^{*}$ & .091 & $.351^{* * * *}$ & .006 \\
\hline & $\begin{array}{l}\text { Spearman } \\
\text { Corr. }\end{array}$ & $.318^{* * *}$ & .012 & $.545^{* * *}$ & .000 & $.509^{* * * *}$ & .000 & $.261^{* *}$ & .044 & $.535^{* * * *}$ & .000 & $.487^{* * * *}$ & .000 \\
\hline & Somers' d & $.207^{* *}$ & .016 & $.370^{* * * *}$ & .000 & $.365^{* * * *}$ & .000 & $.164^{*}$ & .072 & $.378^{* * * *}$ & .000 & $.352^{* * * *}$ & .000 \\
\hline \multicolumn{2}{|c|}{ Independent variable } & DR & & & & & & $\mathrm{PC}$ & & & & & \\
\hline
\end{tabular}




\begin{tabular}{|c|c|c|c|c|c|c|c|c|c|c|c|c|c|}
\hline & $\begin{array}{l}\text { Pearson's } \\
\text { R }\end{array}$ & .176 & .583 & .349 & .267 & .240 & .452 & .448 & .144 & .306 & .333 & .394 & .204 \\
\hline \multirow[t]{4}{*}{ Banks } & Spearman & & & & & & & & & & & & \\
\hline & Corr. & .206 & .520 & .363 & .246 & .246 & .442 & .490 & .106 & $.636^{* *}$ & .206 & $.594^{* *}$ & .042 \\
\hline & Somers' d & .133 & .634 & .267 & .198 & .167 & .507 & $.333^{* *}$ & .040 & $.455^{* * * *}$ & .002 & $.424^{* * *}$ & .019 \\
\hline & $\begin{array}{l}\text { Pearson's } \\
\text { R }\end{array}$ & .233 & .185 & .117 & .508 & .090 & .611 & .161 & .363 & .074 & .679 & .063 & .724 \\
\hline \multirow{4}{*}{$\begin{array}{l}\text { Issuers of } \\
\text { shares }\end{array}$} & Spearman & & & & & & & & & & & & \\
\hline & Corr. & .279 & .110 & .250 & .154 & .078 & .662 & .205 & .244 & .255 & .146 & .049 & .785 \\
\hline & Somers' d & .399 & .203 & .355 & .224 & .115 & .606 & .146 & .470 & .182 & .155 & .059 & .687 \\
\hline & $\begin{array}{l}\text { Pearson's } \\
\text { R }\end{array}$ & .162 & .563 & .297 & .282 & -.170 & .545 & -.138 & .623 & .149 & .596 & -.066 & .816 \\
\hline \multirow[t]{3}{*}{$\begin{array}{l}\text { Insurance } \\
\text { companies }\end{array}$} & $\begin{array}{l}\text { Spearman } \\
\text { Corr. }\end{array}$ & .055 & .847 & .071 & .802 & .022 & .938 & -.257 & .355 & -.246 & .376 & -.279 & .315 \\
\hline & Somers' d & .043 & .843 & .021 & .919 & .043 & .844 & -.181 & .473 & -.219 & .372 & -.162 & .421 \\
\hline & $\begin{array}{l}\text { Pearson's } \\
\text { R }\end{array}$ & $.251^{*}$ & .051 & $.220^{*}$ & .089 & .180 & .166 & .103 & .430 & .153 & .240 & $.259^{* *}$ & .044 \\
\hline \multirow[t]{2}{*}{$\begin{array}{l}\text { All } \\
\text { entities }\end{array}$} & $\begin{array}{l}\text { Spearman } \\
\text { Corr. }\end{array}$ & $.317^{* *}$ & .013 & $.505^{* * * *}$ & .000 & $.309^{* *}$ & .015 & .188 & .147 & $.417^{* * * *}$ & .001 & $.337^{* * * *}$ & .008 \\
\hline & Somers' d & $.301^{* * * *}$ & .006 & $.475^{* * * *}$ & .000 & $.295^{* * * *}$ & .005 & .127 & .135 & $.293^{* * * *}$ & .000 & $.243^{* *}$ & .012 \\
\hline \multicolumn{2}{|c|}{ Independent variable } & TI & & & & & & & & & & & \\
\hline \multirow{3}{*}{ Banks } & $\begin{array}{l}\text { Pearson's } \\
\text { R }\end{array}$ & -.044 & .892 & .350 & .265 & -.002 & .996 & & & & & & \\
\hline & $\begin{array}{l}\text { Spearman } \\
\text { Corr }\end{array}$ & -441 & 152 & 217 & 499 & -182 & 572 & & & & & & \\
\hline & Somers' d & -.303 & .130 & .182 & .483 & -.152 & .474 & & & & & & \\
\hline \multirow{3}{*}{$\begin{array}{l}\text { Issuers of } \\
\text { shares }\end{array}$} & $\begin{array}{l}\text { Pearson's } \\
\mathrm{R}\end{array}$ & -.030 & .867 & $\begin{array}{l}- \\
.342^{* *}\end{array}$ & .048 & .060 & .735 & & & & & & \\
\hline & $\begin{array}{l}\text { Spearman } \\
\text { Corr. }\end{array}$ & -.078 & .662 & -.076 & .670 & -.055 & .755 & & & & & & \\
\hline & Somers' d & -.045 & .718 & -.041 & .757 & -.048 & .762 & & & & & & \\
\hline \multirow{3}{*}{$\begin{array}{l}\text { Insurance } \\
\text { companies }\end{array}$} & $\begin{array}{l}\text { Pearson's } \\
\text { R }\end{array}$ & $\begin{array}{l}- \\
.813^{* * * *}\end{array}$ & .000 & -.395 & .145 & -.261 & .348 & & & & & & \\
\hline & $\begin{array}{l}\text { Spearman } \\
\text { Corr. }\end{array}$ & $\begin{array}{l}- \\
.629^{* * *}\end{array}$ & .012 & -.221 & .428 & $-.468^{*}$ & .079 & & & & & & \\
\hline & Somers' d & - & 009 & -162 & 452 & - & 044 & & & & & & \\
\hline \multirow{3}{*}{$\begin{array}{l}\text { All } \\
\text { entities }\end{array}$} & $\begin{array}{l}\text { Pearson's } \\
\text { R }\end{array}$ & .066 & .619 & -.090 & .488 & $.233^{*}$ & .071 & & & & & & \\
\hline & $\begin{array}{l}\text { Spearman } \\
\text { Corr. }\end{array}$ & -.091 & .484 & .203 & .117 & $.217^{*}$ & .093 & & & & & & \\
\hline & Somers' d & -.090 & .375 & .149 & .118 & .158 & .127 & & & & & & \\
\hline
\end{tabular}

*Correlation is significant at the 0.1 level (2-tailed). **Correlation is significant at the 0.05 level (2-tailed). ***Correlation is significant at the 0.01 level (2-tailed).

Source: own calculations based on data from annuals reports of individual companies 


\section{Results}

The author examined the influence of selected determinants of CG on a sample of companies that form a basic set in the conditions of Slovakia.

The first group of entities consisted of banks and building societies. As of December 31, 2017, according to the NBS data, nine banks, three home savings banks and fourteen branches of foreign banks operated in the Slovak Republic. The longest operating banks on the Slovak market are the VUB bank and the Tatra bank, founded in 1990. The youngest bank is ČSOB, founded in 2008. The highest profitability in the period under review was achieved by Privatbanka, while the OTP bank's economic result and profitability were both negative. SLSP achieved the highest net profit in 2017. Six banks are owned by only one institutional investor; in the other four banks the largest shareholder owns at least $89 \%$ of the registered capital. VUB has the largest volume of equity, followed by SLSP. ČSOB has the least equity. Five of the banks paid more than $90 \%$ of the 2016 net profit to their shareholders in 2017, while four of the banks paid out no dividends at all. The highest personnel costs per employee were reported by Privatbanka, the lowest by the Wüstenrot home savings bank. The highest remuneration per member of the governing bodies was paid by the Post Bank, the lowest by the SZRB (Slovak Guarantee and Development Bank). Further descriptive statistics of the variables are given in Table 3.

Table 3: Descriptive statistics of variables

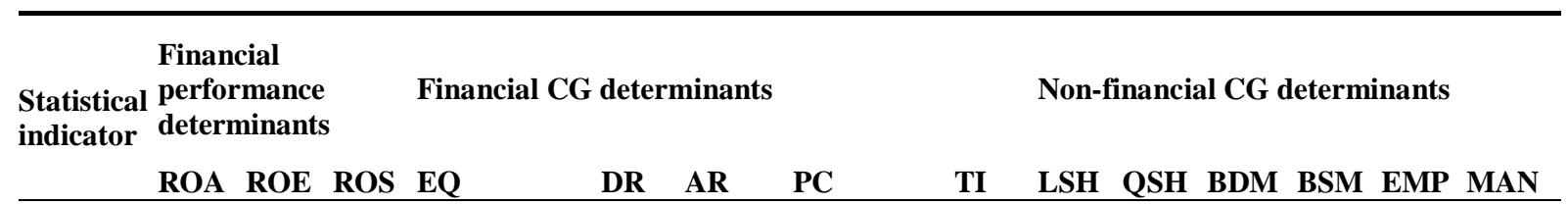

All entities (number: 61)

$\begin{array}{lllllllllllllll}\text { Mean } & 0.00 & -0.03 & 0.06 & 147,094,785 & 0.33 & 61,944 & 14,409,559 & 0.61 & 0.69 & 2 & 4 & 5 & 494 & 0.08 \\ \text { Median } & 0.01 & 0.05 & 0.07 & 18,454,098 & 0.00 & 19,533 & 3,335,483 & 0.68 & 0.80 & 2 & 3 & 3 & 160 & 0.05 \\ \text { Std. Dev. } & 0.07 & 0.52 & 0.24 & 360,718,882 & 0.47 & 82,480 & 29,899,101 & 0.33 & 0.31 & 1 & 1 & 2 & 902 & 0.09 \\ \text { Skewness } & -3.92 & -5.61 & -0.60 & 3 & 1.07 & 1 & 3 & -0.15 & -0.50 & 1 & 1 & 2 & 3 & 1.40 \\ \text { Kurtosis } & 20.90 & 35.80 & 3.82 & 10 & -0.13 & 1 & 8 & -0.39 & -1.39 & 1 & 3 & 2 & 8 & 1.24 \\ \text { Minimum } & -0.42 & -3.53 & -0.81 & -732,318 & 0.00 & 0 & 0 & 0.00 & 0.17 & 1 & 1 & 2 & 0 & 0.00 \\ \text { Maximum } & 0.11 & 0.67 & 0.73 & 1,599,689,000 & 1.70 & 321,111 & 133,399,000 & 1.52 & 1.00 & 7 & 9 & 13 & 4208 & 0.33 \\ \text { Insurance companies (number: } \mathbf{1 5}) & & & & & & & & & & \\ \text { Mean } & 0.03 & 0.11 & 0.11 & 77,350,533 & 0.60 & 78,000 & 10,677,200 & 0.80 & 0.93 & 2 & 4 & 5 & 396 & 0.12 \\ \text { Median } & 0.02 & 0.12 & 0.08 & 47,760,000 & 0.85 & 56,727 & 6,495,000 & 0.83 & 1.00 & 2 & 4 & 4 & 298 & 0.13 \\ \text { Std. Dev. } & 0.03 & 0.11 & 0.20 & 96,790,802 & 0.46 & 57,752 & 12,525,258 & 0.10 & 0.09 & 1 & 1 & 3 & 442 & 0.07 \\ \text { Skewness } & 1.57 & -1.19 & 2.33 & 2 & -0.51 & 2 & 2 & -1.38 & -1.05 & 1 & 0 & 1 & 2 & 0.42 \\ \text { Kurtosis } & 3.69 & 2.42 & 8.63 & 3 & -1.59 & 5 & 5 & 1.75 & -0.30 & 0 & 1 & 1 & 3 & -0.47 \\ \text { Minimum } & -0.01 & -0.18 & -0.21 & 8,170,000 & 0.00 & 25,333 & 1,301,000 & 0.54 & 0.73 & 1 & 2 & 2 & 36 & 0.01 \\ \text { Maximum } & 0.11 & 0.27 & 0.73 & 304,709,000 & 1.23 & 247,000 & 47,130,000 & 0.93 & 1.00 & 3 & 7 & 13 & 1516 & 0.25\end{array}$

Non-financial corporations listed on stock exchange (issuers of shares) (number: 34)

$\begin{array}{lllllllllllllll}\text { Mean } & -0.01 & -0.15 & -0.01 & 57,725,555 & 0.14 & 18,157 & 5,392,473 & 0.43 & 0.49 & 3 & 3 & 4 & 204 & 0.08 \\ \text { Median } & 0.00 & 0.00 & 0.01 & 3,089,196 & 0.00 & 2,424 & 766,841 & 0.39 & 0.41 & 3 & 3 & 3 & 44 & 0.04 \\ \text { Std. Dev. } & 0.09 & 0.67 & 0.25 & 273,391,249 & 0.39 & 46,721 & 17,797,822 & 0.33 & 0.28 & 1 & 1 & 1 & 455 & 0.10\end{array}$




\begin{tabular}{|c|c|c|c|c|c|c|c|c|c|c|c|c|c|c|}
\hline Skewness & -3.05 & -4.47 & -0.72 & 6 & 3.17 & 3 & 5 & 1.17 & 0.45 & 1 & 1 & 2 & 4 & 1.69 \\
\hline Kurtosis & 11.83 & 21.42 & 3.44 & 33 & 9.76 & 12 & 30 & 2.05 & -1.27 & 1 & 4 & 5 & 16 & 1.73 \\
\hline Minimum & -0.42 & -3.53 & -0.81 & -732318 & 0.00 & 0 & 0 & 0.00 & 0.17 & 1 & 1 & 3 & 0 & 0.00 \\
\hline Maximum & 0.08 & 0.23 & 0.68 & $1,599,689,000$ & 1.70 & 220,462 & $103,001,000$ & 1.52 & 1.00 & 7 & 7 & 9 & 2,341 & 0.33 \\
\hline \multicolumn{15}{|c|}{ Banks (number: 12) } \\
\hline Mean & 0.01 & 0.11 & 0.21 & $487,487,917$ & 0.51 & 162,285 & $44,623,417$ & 0.87 & 0.90 & 2 & 5 & 6 & 1,440 & 0.05 \\
\hline Median & 0.01 & 0.07 & 0.22 & $266,174,000$ & 0.55 & 178,876 & $18,223,500$ & 0.90 & 1.00 & 1 & 4 & 6 & 808 & 0.03 \\
\hline Std. Dev. & 0.00 & 0.18 & 0.15 & $562,016,643$ & 0.45 & 93,843 & $49,598,309$ & 0.13 & 0.21 & 1 & 2 & 3 & 1,523 & 0.05 \\
\hline Skewness & -0.76 & 3.01 & -1.82 & 1 & -0.11 & 0 & 1 & -3.07 & -2.42 & 2 & 1 & 1 & 1 & 1.48 \\
\hline Kurtosis & 1.32 & 9.89 & 5.00 & 0 & -2.14 & -1 & -1 & 10.21 & 5.59 & 5 & 1 & -1 & -1 & 0.84 \\
\hline Minimum & -0.00 & -0.05 & -0.19 & $25,962,000$ & 0.00 & 9,214 & $2,892,000$ & 0.46 & 0.33 & 1 & 3 & 3 & 121 & 0.01 \\
\hline Maximum & 0.01 & 0.67 & 0.40 & $1,555,980,000$ & 1.00 & 321,111 & $133,399,000$ & 0.99 & 1.00 & 5 & 9 & 11 & 4,208 & 0.14 \\
\hline
\end{tabular}

Source: own calculations based on data from annuals reports of individual companies

The second group of examined subjects were insurance companies. As of December 31, 2017, according to the NBS data, a total of sixteen insurance companies based in Slovakia and twenty-six branches of foreign insurance companies were operating in the Slovak Republic. One insurance company declared bankruptcy in January 2018, which is why it was excluded it from the set of researched companies and a total of fifteen insurance companies was examined. The largest insurance company in Slovakia in terms of equity is Kooperativa, followed by Allianz; the same companies were the top two according to the amount of net profit achieved in 2017, in reversed order. Ergo has the lowest equity and reported the highest loss in 2017. The Union insurance company reported a loss too. A total of six insurance companies are owned by one shareholder, in the other four the main shareholder holds more than $90 \%$ of the registered capital. Kooperativa had the highest number of members of the boards. The highest average personnel costs per employee were reported by the Union insurance company, the lowest by the insurance company NN, followed by the Wüstenrot insurance company. The highest ROE was reported by Cardif, while a negative ROE was reported by ERGO. Generali reported the lowest ROE among the profitable insurance companies, but paid out the second highest average remuneration (AR) per member of the governing bodies of the insurance companies involved. The highest AR was paid by Allianz, the lowest by Poštová poist'ovňa. The highest share of dividends paid from the previous year's profit was reported by Aegon. A total of seven insurance companies paid at least $90 \%$ of their net profit to their shareholders. Five insurance companies, including Allianz, state in their annual report that they did not pay any dividends in 2017.

The third group of examined entities were joint-stock companies, share issuers, listed on the Bratislava Stock Exchange ("companies"). Companies that issued only bonds were not examined. As of December 31, 2017, thirty-eight issuers of shares were listed on the Bratislava Stock Exchange, of which there was one insurance company (Union), thee banks (the Tatra bank, the VÚB bank, the Prima bank) and thirty-four non-financial joint-stock companies; the focus was on this latter group of entities. The largest company is Slovnaft. In 2017, this company reported the highest volume of assets, equity, and net profit; it is the employer with the highest number of employees and the company with the second highest average personnel costs per employee. It however ranked sixth in ROE, and fifth in ROA. Slovnaft paid the highest average remuneration per member of the governing bodies. A total of twelve companies, including ZTS INMART, which achieved the highest ROE in this group of entities and did not employ any employees, did not pay any remuneration to the governing bodies. The highest average personnel costs per employee are reported by Geocomplex, which reported 1.36 
percentage points higher ROA and $20.2 \mathrm{pp}$. higher ROS than Slovnaft. Slovnaft, compared to Geocomplex, reached a $1.79 \mathrm{pp}$. higher ROE and paid 31.5 times higher remuneration per member of the governing bodies.

Capital strength of a company allows payment of higher remuneration to the governing bodies, even though the relative economic results may not be among the best. From a quantitative point of view, this finding is also confirmed by the results of the correlation analysis of AR dependence and financial performance indicators measured by Pearson's $r$ (see Table A1). Based on Sig. it can be argued that there is no correlation between financial performance and the average level of remuneration per person of governing bodies in any of the groups of entities under review. The existence of dependence between AR as a dependent variable and EQ as an independent variable was confirmed by a Somers' D test in all groups of subjects and in the entire set of subjects. A small dependence of AR on EQ was found in banks ( $r=0.273$, Sig. $=0.066)$, a moderate dependence in the group of companies $(r=0.470$, Sig. $=0.000)$, and a large dependence in insurance companies $(\mathrm{r}=0.562$, Sig. $=0.000)$ as well as in the entire set of subjects $(r=0.678$, Sig. $=0.000)$.

In accordance with the objectives and selected methods, the researcher tested and compared the impact of selected determinants of CG on the financial performance of entities. In the text, the statistically significant results are interpreted, ascertained by Somers' D, on the selected significance level. Other results are shown in Table A1.

In the first step the influence of financial and non-financial determinants of CG on ROA was evaluated. A moderate negative correlation was found between ROA and TI $(r=-0.467)$ in insurance companies. Other subjects did not show a statistically significant effect of TI on ROA. The results indicate moderate dependence between ROA and BSM in companies $(r=0.451)$; between ROA and EQ $(r=0.364)$, ROA and PC $(r=0.333)$ in banks. Weak dependence was identified between ROA and AR $(r=0.299)$, ROA and EQ $(r=0.294)$ in companies. In the group of all companies, a moderate correlation was found between ROA and DR $(r=0.301)$, between ROA and EQ $(r=0.207)$, between ROA and BSM $(r=0.195)$, and between ROA and $\operatorname{AR}(r=0.164)$.

When examining the influence of $\mathrm{CG}$ determinants on ROE, significant results were found only in the group of companies and banks. In banks, it was between ROE and PC $(r=0.455)$, AR $(r=0.424)$, and EMP $(r=0.424)$. In companies, it was between ROE and BSM $(r=0.487)$, EQ $(r=0.333)$ and AR $(r=0.320)$. All values show moderate dependence of variables. In the group of all entities, moderate dependence was revealed between ROE and DR $(r=0.475)$, ROE and AR $(r=0.378)$, ROE and EQ $(r=0.370)$, ROE and BSM $(r=0.301)$; weak dependence between ROE and PC ( $r=0.293)$, ROE and EMP $(r=0.261)$ and ROE and LSH $(r=0.208)$.

Moderate influence of EQ $(r=0.515)$, PC $(r=0.424)$, and EMP $(r=0.333)$ on ROS was found in the group of banks. In the insurance companies, a moderate negative correlation was calculated between ROS and TI $(r=-0.333)$. All values are significant at the selected significance level. A weak dependence was unveiled in the group of companies between ROS and BSM $(r=0.278), A R(r=0.238)$ and EQ $(r=0.219)$. In the group of all subjects, moderate correlation was found between ROS and EQ $(r=0.365)$, AR $(r=0.352)$, DR $(r=0.295)$, PC $(r$ $=0.243), \operatorname{BSM}(r=0.229)$ and $\operatorname{EMP}(r=0.218)$.

\section{Discussion}

Corporate governance is currently one of the most widely discussed topics and its interactions with various areas and levels of business activities are an object of research. Ortas et al. (2015) examine the impact of institutional and social context on corporate environment, 
social and governance performance of enterprises. The role of corporate governance in relation to corporate social responsibility and financial performance has been explored by e.g. Rodrigues-Fernandez (2016), Broadstock et al. (2019), Musa et al. (2014a). Gender equality and diversity, corporate performance, and emotional intelligence in relation to corporate governance were examined by Báez et al. (2018), Musa et al. (2017). Corporate governance compliance for family and non-family firms in emerging markets was examined by BrianoTurrent and Poletti-Hughes (2017). Corporate governance in emerging markets has also been investigated e.g. by Bhaumik et al. (2019), Koirala et al. (2018), and Esqueda and O'Connor (2019).

In terms of long-term financial prosperity of business entities, the most important research is that of the impact of corporate governance on financial management and decision-making processes and the relevant financial risks. Ali et al. (2018) or Shahid and Abbas (2019) examined whether the quality of corporate governance affects the risk of business failure, or the role corporate governance plays in relation to investors and investment decisions.

Also examined in this paper is the impact of corporate governance on the financial performance and prosperity of the company. The chosen determinants are divided according to their character into financial and non-financial. Financial determinants of corporate governance that significantly affect corporate performance as measured by the ROA, ROE, and ROS indicators include EQ, DR, AR, and PC. A significant non-financial determinant is BSM. From the results, it is possible to identify several financial determinants of corporate governance, which should be considered in business management and decision-making processes. We did not find any research using the same or similar methodology for the classification of variables and business entities, which is why the present paper can be considered a contribution to the development of knowledge in the field of corporate governance.

\section{Conclusion}

To summarize the findings of the research, it can be concluded that each of the investigated financial and non-financial determinants of $\mathrm{CG}$ affects financial performance of companies. At the same time, ROA, ROE, and ROS are influenced in the group of share issuers by the amount of equity (EQ), AR, and BSM; and PC in banks. In the group of all enterprises, they are influenced by EQ, DR, AR, and BSM.

The EQ indicator in banks and the TI indicator in insurance companies have an influence on two performance indicators (ROA and ROS) at the same time. BSM affects both ROA and ROE (at the same time) in the group of companies.

By evaluating the impact of CG determinants on only one of the performance indicators, the research found an effect of EQ on ROA in banks, TI on ROA in insurance companies; an impact of AR, EMP and MAN on ROE in banks and TI on ROE in enterprises; an impact of EQ, EMP and PC on ROS in banks.

ROA is most strongly negatively influenced by TI in insurance companies (Pearson's $r=-$ 0.813, Sig. $=0.000)$, the highest positive correlation was found between ROA and BSM in companies (Somers' $\mathrm{D}=0.451$, Sig. $=0.016$ ). ROE in banks is most strongly influenced by the MAN indicator (Pearson's $r=0.597$, Sig. $=0.089$ ); ROS in banks is most strongly influenced by the EQ indicator (Spearman $r=0.608$, Sig. $=0.036$ ). 


\section{Acknowledgment}

The authors would like to express their gratitude to the Scientific Grant Agency of The Ministry of Education, Science, Research and Sport of the Slovak Republic for financial support of this research and publication.

This research was funded by the Scientific Grant Agency of Slovak Republic under project VEGA No. 1/0749/18, ,Research on the application of CG principles in companies in Slovakia“.

\section{References}

Afza, T., \& Nazir, M. S. (2014). Theoretical perspective of corporate governance: A review. European Journal of Scientific Research, 119(2), 255-264.

Akbar, S., Poletti-Hughes, J., El-Faitouri, R., Zulfiqar, S., \& Shah, A. (2016). More on the relationship between corporate governance and firm performance in the UK: Evidence from the application of generalized method of moments estimation. Research in International Business and Finance, 38, 417-429.

Ali, S. Liu, B., \& Su, J. J. (2018). Does corporate governance quality affect default risk? The role of growth opportunities and stock liquidity. International Review of Economics and Finance, 58(2018), 422-448.

Ararat, M., Black, B. S., \& Yurtoglu, B. B. (2017). The effect of corporate governance on firm value and profitability: Time-series evidence from Turkey. Emerging Markets Review, 30, 113-132.

Báez, A. B., Báez-García, A. J., Flores-Muñoz, F., \& Gutiérrez-Barroso, J. (2018). Gender diversity, corporate governance and firm behavior: The challenge of emotional management. European Research on Management and Business Economics, 24(2018), 121-129.

Bhagat, S., \& Bolton, B. (2008). Corporate governance and firm performance. Journal of Corporate Finance, 14(3), 257-273.

Bhagat, S., \& Bolton, B. (2019). Corporate governance and firm performance: The sequel. Journal of Corporate Finance, 58(2019), 142-168.

Bhaumik, S., Driffield, N., Gaur, A., Mickiewicz, T., \& Vaaler, P. (2019). Journal of World Business, 54(2019), 234-243.

Briano-Turrent, G. del C., \& Poletti-Hughes, J. (2017). Corporate governance compliance of family and nonfamily listed firms in emerging markets: Evidence from Latin America. Journal of Family Business Strategy, 8(2017), 237-247.

Broadstock, D. C., Matousek, R., Meyer, M., \& Tzeremes, N. G. (2019). Does corporate social responsibility impact firms' innovation capacity? The indirect link between environmental and social governance implementation and innovation performance. Journal of Business Research, 1-12.

Detthamrong, U., Chancharat, N., \& Vithessonthi, Ch. (2017). Corporate governance, capital structure and firm performance: Evidence from Thailand. Research in International Business and Finance, 42(2017), 689-709.

Di Berardino, D. (2016). Corporate governance and firm performance in new technology ventures. Procedia Economics and Finance. 39(2016), 412-421.

Esqueda, O. A., \& O'Connor, T. (2019). Corporate governance and life cycle in emerging markets. Research in International Business and Finance, 51(2020), 1-24.

Fooladi, M., Zaleha, A. S., Norman, M. S., \& Romlah, J. (2014). The effect of corporate governance and divergence between cash flow and control rights on firm performance: Evidence from Malaysia. International Journal of Disclosure and Governance, 11(4), 326-340. 10.1057/jdg.2013.24.

Grofcikova, J. (2016a). Globalization and its impact on the corporate financial management and control. In: T. Klieštik (Ed.) Proceedings of the 16th International Scientific Conference on Globalization and Its SocioEconomic Consequences. Rajecké Teplice: University of Žilina, Slovak Republic, 547-555.

Grofcikova, J. (2016b). The use of financial analysis indicators for the monitoring of company financial situation and elimination of financial risk. In: M. Čulík (Ed.) Proceedings of the 8th international scientific conference on Managing and Modelling of Financial Risks. Ostrava: Technical University, Czech Republic, 248-255.

Hearn, B. (2011). The impact of corporate governance measures on the performance of West African IPO firms. Emerging Markets Review, 12(2011), 130-151.

Khanchel, I. (2007). Corporate governance: Measurement and determinant analysis. Managerial Auditing Journal, 22(8), 740-760.

Koirala, S., Marshall, A., Neupane, S., \& Thapa Ch. (2018). Corporate governance reform and risk-taking: Evidence from a quasi-natural experiment in an emerging market. Journal of Corporate Finance, 1-22.

Mizuno, M. (2010). Institutional investors, corporate governance and firm performance in Japan. Pacific Economic Review, 15(5), 653-665. 
Musa, H., Debnarova, L., Musova, Z., \& Kristofik, P. (2017). Gender equality and corporate governance in Slovakia. E+M. Ekonomie a Management, 20(1), 98-100.

Musa, H., Kristofik, P., \& Debnarova, L. (2014a). Corporate governance and its impact on financial risk. In: M. Culik (Ed.) Proceedings of the 7th International Scientific Conference on Managing and Modelling of Financial Risks. Ostrava: Technical University, Czech Republic, 529-535.

Musa, H., Musova, Z., \& Debnarova, L. (2014b). Responsibility in the corporate governance framework and financial decision making process. In: Procedia Economics and Finance: 2nd Global Conference on Business, Economics, Management and Tourism. Prague, Czech Republic, 1023-1029.

Naciti, V. (2019). Corporate governance and board of directors: The effect of a board composition on firm sustainability performance. Journal of Cleaner Production, 237(2019), 1-8.

Ortas, E., Álvarez, I., Jaussaud, J., \& Garayar, A. (2015). The impact of institutional and social context on corporate environmental, social and governance performance of companies committed to voluntary corporate social responsibility initiatives. Journal of Cleaner Production, 108(2015), 673-684.

Paniagua, J., Rivelles, R., \& Sapena, J. (2018). Corporate governance and financial performance: The role of ownership and board structure. Journal of Business Research, 89(2018), 229-234.

Rodriguez-Fernandez, M. (2016). Social responsibility and financial performance: The role of good corporate governance. BRQ Business Research Quarterly, 2016(19), 137-151.

Schymik, J. (2018). Globalization and the evolution of corporate governance. European Economic Review, 102(2018), 39-61.

Shahid, M. S., \& Abbas, M. (2019). Does corporate governance play any role in investor confidence, corporate investment decisions relationship? Evidence from Pakistan and India. Journal of Economics and Business, 105(2019), 1-11.

Shleifer, A., \& Vishny, R. W. (1997). A survey of corporate governance. The Journal of Finance, 52(2), 737-783.

The Organisation for Economic Co-operation and Development. Retrieved from http://www.oecd.org/.

Valaskova, K., Kliestik, T., \& Kovacova, M. (2018). Management of financial risks in Slovak enterprises using regression analysis. Oeconomia Copernicana, 9(1), 105-121.

Vo, H. D., \& Phan, B. G. T. (2013). Corporate governance and firm performance: Empirical Evidence from Vietnam. Journal of Economic Development, 218, 62-77.

Vu, N. H., \& Nguyen, T. (2017). Impacts of corporate governance on firm performance. Empirical study of listed Singaporean companies. [Online]. Lund University, 1-66. Retrieved from http://lup.lub.lu.se/luur/download?func=downloadFile\&recordOId=8917364\&fileOId=8917365.

Waleed, M. A., Mohammed, H. A., Mosab, I. T., \& Najib, H. S. F. (2019). The impact of corporate governance on financial performance of Indian and GCC listed firms: An empirical investigation. Research in International Business and Finance, 51(2020), 1-13. doi: 10.1016/j.ribaf.2019.101083.

Zahroh, N., \& Hamidah. (2016). The role of corporate governance in firm performance. SHS Web of Conferences, 34(13003), 1-6. 